\title{
CREating a SCAP-less liver keeps SREBPs pinned in the ER membrane and prevents increased lipid synthesis in response to low cholesterol and high insulin
}

\author{
Timothy F. Osborne ${ }^{1}$ \\ Department of Molecular Biology and Biochemistry, University of California, Irvine, California 92697, USA
}

Cellular cholesterol homeostasis in mammals is governed by a small subfamily of bHLHLZ transcription factors called the sterol regulatory element binding proteins (SREBPs). When they were first identified in 1993 (Hua et al. 1993; Tontonoz et al. 1993; Yokoyama et al. 1993), the significance of the amino-terminal bHLHLZ domain as a transcriptional regulatory protein was obvious because of its similarity with other proteins such as Myc and Max. However, because the remaining $50 \%$ of the predicted protein sequence was not similar to any other known proteins, its significance was not appreciated. Elegant studies over the ensuing years have shown that the SREBPs are synthesized as precursor proteins that are threaded into the endoplasmic reticulum (ER) membrane and anchored there through a two-pass membrane-spanning domain (Brown and Goldstein 1999). The two membrane domains pin the inactive precursor in a rigid hairpin orienting both the amino- and carboxy-terminal domains to the cytosolic face of the membrane. SREBPs are maintained in this inactive form until a low sterol level is sensed and they are needed to increase lipid accumulation. The low sterol level sets a proteolytic cascade in motion that results in nuclear accumulation of an amino-terminal fragment that corresponds to the mature SREBP transcriptional regulatory protein.

Key steps in the mechanism for the sterol-regulated cleavage process have been revealed through a classic combination of somatic cell-molecular genetics and biochemistry (Brown and Goldstein 1999). Three additional membrane-bound proteins are involved in the multistep regulation in addition to the SREBPs themselves. These include two proteases (Rawson et al. 1997; Sakai et al. 1998) and a polytopic ER membrane protein with a putative sterol-sensing domain (Hua et al. 1996a), which is

${ }^{1}$ E-MAIL tfosborn@uci.edu; FAX (949) 824-8551.

Article and publication are at http://www.genesdev.org/cgi/doi/10.1101/ gad.916601. involved in modulating access of the substrate SREBPs with the first protease. Although the need for proteolysis was obvious, the role of the regulatory protein, called SREBP cleavage activation protein or SCAP, was not apparent. It is now clear that SCAP is the key to sterol regulation because the identical proteases, but not SCAP, are involved in releasing the ATF 6 protein from ER membranes in response to cellular stress (Ye et al. 2000). In addition, a complex between SCAP and SREBPs moves from the ER to the golgi in a sterol-regulated manner (Nohturfft et al. 1999, 2000). This places the substrate SREBPs into the same cellular compartment as the proteases (Fig. 1). Thus, the role of SCAP is to escort the SREBPs from one cellular compartment to another in a sterol-regulated fashion

Sheng et al. (1995) showed that processing of SREBP-1 and SREBP-2 is regulated differently in animals, whereas their processing is coordinately regulated in cultured cells in response to sterol depletion (Wang et al. 1994; Hua et al. 1996b). SCAP is required for maturation of both SREBP-1 and SREBP-2 isoforms in cultured cells, but because of the results of Sheng et al. (1995) there was a question as to whether SCAP was really involved in processing both SREBP-1 and SREBP-2 in animals. Recently, Matsuda et al. (2001) used Cre-loxP gene knockout technology combined with animal feeding studies to evaluate SCAP's role in processing precursor SREBPs in livers of adult mammals. Loss of liver SCAP resulted in severely reduced levels of mRNAs and precursor proteins for both SREBP-1 and SREBP-2 in livers of animals fed a standard rodent balanced chow diet. Thus, SCAP is definitely involved in maturation of both SREBPs, but there is surely more to be uncovered in this system because of the differential regulation noted by Sheng et al. (1995). In the first part of this article, I provide a brief overview to the SREBP pathway and refer the reader to other recent reviews of this more general topic (Brown and Goldstein 1999; Edwards and Ericsson 1999; Edwards et al. 2000; Osborne 2000). The second part of the 


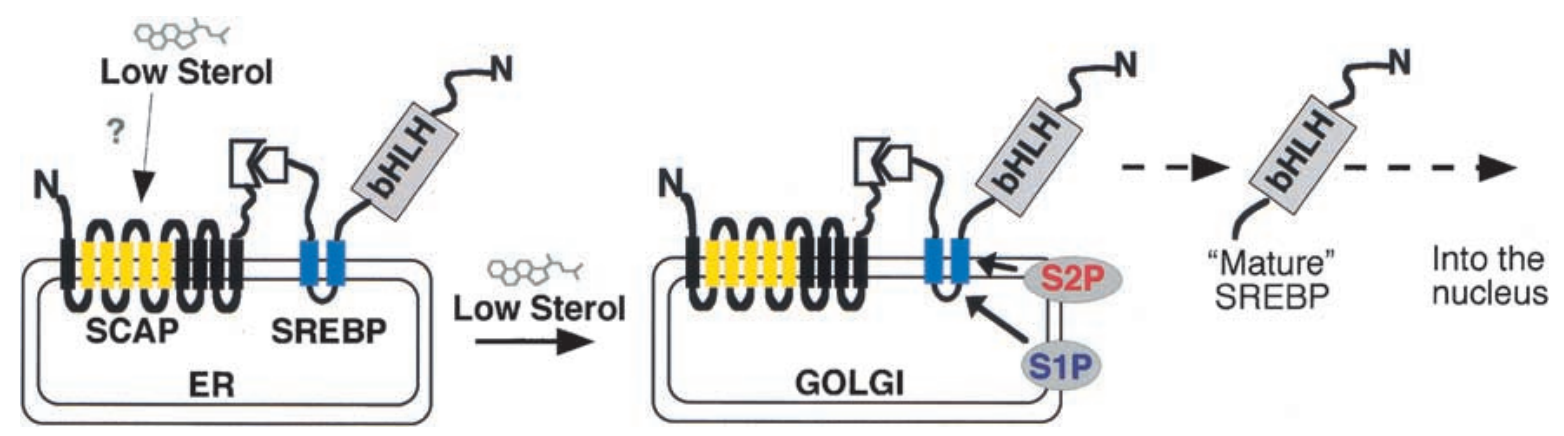

Figure 1. SCAP escorts SREBPs from the endoplasmic reticulum (ER) to Golgi in response to low sterol levels. Both SCAP and SREBP are shown anchored to the ER membrane through several or two membrane-spanning domains, respectively. The putative sterolsensing domain of SCAP, indicated in yellow, represents a membrane-spanning region with similarities to several other proteins that are likely to be involved in sterol sensing in the cell (Brown and Goldstein 1999). An interaction between the carboxy-terminal domains of the two proteins is also indicated. Under low sterol conditions, the SCAP-SREBP complex travels to the Golgi complex in which the two sequential acting proteases, S1P and S2P, reside. After cleavage by S2P, the mature transcription factor enters the nucleus.

article is focused on SCAP with an emphasis on the significance of the study from Matsuda et al. (2001).

\section{The SREBP family: key regulators of lipid metabolism}

The SREBPs were identified independently by two different groups working on adipocyte cell differentiation (Tontonoz et al. 1993) and cholesterol metabolism (Hua et al. 1993; Yokoyama et al. 1993). DNA sequence analyses revealed that SREBPs are members of the bHLHLZ family of transcription factors, but they have two special properties that distinguish them from other family members. First, they are translated as membrane-bound precursors as mentioned above. The second special feature is a unique tyrosine residue in the basic region of the bHLH motif (Kim et al. 1995). All other related bHLH proteins that have been identified to date, with the exception of two yeast proteins, have an arginine at the equivalent position. Interestingly, one of the yeast proteins has been implicated in controlling carbohydrate catabolism (Nishi et al. 1995) and the other is involved in pseudohyphal growth regulation (Lane et al. 2001). Because carbohydrate breakdown is a fundamental part of lipogenesis and pseudohyphal growth requires increased accumulation of lipids for membrane expansion, it is conceivable that regulation of these two processes in yeast is related through evolution to regulation of key SREBP-dependent processes in mammals. The two yeast proteins do not share the carboxy-terminal extension that anchors the SREBPs into membranes.

The tyrosine residue allows alternative folding of the DNA-binding domain, permitting high-affinity recognition of two very different DNA sites (Parraga et al. 1998). One site is the inverted repeat E-box, which is the signature DNA recognition site of bHLH proteins. The other site is the direct repeat sterol regulatory element (SRE) (Kim et al. 1995), which is present in all known sterol-regulated genes identified to date (Athanikar and Osborne 1998). The functional implications of this dual DNA-binding specificity are discussed elsewhere (Os- borne 2000). Interestingly, the two yeast proteins also bind to both classes of DNA sites.

There are two SREBP genes and three major proteins in the SREBP clan. Two transcripts are produced from the SREBP-1 gene by use of two different promoters (Miserez et al. 1997). Transcription from each promoter produces an SREBP-1 mRNA with a unique first exon that is ligated to a common second exon during mRNA processing. The mRNA reading frame into the second exon is the same for both transcripts, so the protein coding sequences for both isoforms are identical from this point forward. Only one mRNA and protein have been identified from the SREBP-2 gene. Important questions include how the different isoforms regulate overlapping but not identical gene sets and how expression and activity of the SREBPs themselves are regulated in similar and distinct ways.

The SREBP transcriptional activation domain is located at the extreme amino terminus, therefore, the alternative promoters in SREBP-1 result in two proteins with different activation domains (Shimano et al. 1997). The longer amino-terminal region in SREBP-1a is a very potent transcriptional activation domain. The SREBP-1c isoform lacks 29 amino acids present in SREBP-1a and has four unique amino acids in addition to the initiator methionine. The shorter amino terminus of SREBP-1c is a very poor activation domain.

The ratio of SREBP-1a and SREBP-1c mRNA levels vary over two orders of magnitude in different tissues of the body. For example, in liver, in which SREBP activity is most significant to nutrient metabolism, SREBP-1c mRNA is close to 10-fold more abundant than SREBP-1a, whereas in spleen, this ratio is reversed (Shimomura et al. 1997). It is not yet clear whether these ratios reflect similar differences in the levels of each protein. In contrast, SREBP-1a mRNA is expressed at higher levels than SREBP-1c in all but one cell line characterized so far (Shimomura et al. 1997; DeBose-Boyd et al. 2001). Because it is encoded by the predominant mRNA in adult liver and adipose tissue, the SREBP-1c protein is likely to 
represent the key protein involved in SREBP-1-dependent processes in these tissues. A significant role for SREBPs in both lipogenesis and cholesterol metabolism has been established convincingly through both genetic manipulation of SREBP levels and feeding studies in animals (for review, see Osborne 2000). From expression studies of individual isoforms, it appears that SREBP-1 preferentially activates genes of fatty acid metabolism, whereas SREBP-2 selectively stimulates genes involved in cholesterol homeostasis (Horton et al. 1998b). An mRNA expression profile of SREBP-1 versus SREBP-2 reveals that they are both expressed widely throughout the body at roughly similar levels with significant overlap (Hua et al. 1993; Yokoyama et al. 1993).

Feeding rodents a high-carbohydrate diet after a forced fast activates the entire program of lipogenesis in liver. This represents a classic insulin response (Towle et al. 1997), and SREBP-1c mRNA was also activated by this protocol (Horton et al. 1998a; Kim et al. 1998). Overexpression of SREBP-1c in livers prevented down-regulation of lipogenic genes that occurs normally during fasting (Horton et al. 1998a). In a related report, a knockout of the SREBP-1 gene resulted in a total loss of lipogenic gene activation during the refeeding phase even though SREBP-2 levels were normal or perhaps slightly elevated (Shimano et al. 1999). These and additional observations discussed in another recent review (Osborne 2000) provide strong evidence that SREBP-1c is a key regulator of early events in the liver's response to insulin.

\section{Sterol-dependent proteolysis of SREBP: no eSCAPing fate}

The studies referred to thus far have highlighted the special properties of SREBPs after they are released from their membrane anchor. The cholesterol-sensitive proteolytic maturation represents an additional area of complexity in which multiple proteins function sequentially, culminating in the release of SREBP from the membrane (Fig. 1). A carboxy-terminal domain in SCAP interacts directly with a carboxy-terminal region of the SREBPs (Sakai et al. 1997), and under steroldepleted conditions, the SCAP-SREBP complex transits from the ER to the golgi (Nohturfft et al. 1999). Through this sterol-regulated movement, the SREBPs are brought into close proximity to the first of two sequentially acting proteases, site 1 protease $(\mathrm{S} 1 \mathrm{P})$, which is a membrane-bound serine protease (Sakai et al. 1998) that attacks a peptide bond in the lumenal loop of the hairpin (Duncan et al. 1997). The resulting hydrolytic cleavage splits SREBP in two, leaving roughly each half attached to the membrane through a separate transmembrane domain.

Once in this form, the amino-terminal half functions as a substrate for a second distinct protease called site 2 protease (S2P). S2P is a membrane-bound zinc-metalloprotease (Rawson et al. 1997) that releases the aminoterminal SREBP fragment from the membrane by cleaving a peptide bond located at an intramembrane site in close proximity to the cytosolic face of the membrane surface (Duncan et al. 1998). The released fragment represents the mature SREBP transcriptional regulatory protein and it migrates to the nucleus and activates appropriate target genes. If the cell sterol level once again rises above a critical level, exodus of the SCAP-SREBP complex from the ER is stopped and further SREBP maturation is switched off. Nuclear SREBPs turn over quickly (Wang et al. 1994), thus, when membrane release is inhibited, SREBP target gene expression returns rapidly to basal levels. This provides a dynamic regulatory mechanism to prevent overaccumulation of cellular lipids. The fate of the carboxy-terminal half that is left in the membrane has not been addressed.

In the experiments of Matsuda et al. (2001), animals containing synthetic lox $P$ sites flanking the first exon of the SCAP gene were crossed to another mouse line that expresses Cre recombinase in response to interferon only in the liver. Following activation of Cre, lox $P$-dependent recombination excised $>90 \%$ of the targeted SCAP DNA from adult liver and resulted in a virtual elimination of the corresponding protein. Importantly, there was no effect on SCAP DNA or protein observed in any other tissue. For the first time, this permitted the chance to evaluate the role of SCAP in the regulation of liver metabolism in an adult mammal.

Loss of SCAP decreased processing of membranebound forms of both SREBP-1 and SREBP-2 proteins in livers of animals fed a normal chow diet. SREBP target gene expression in liver was also reduced as were serum and liver levels of triglycerides and cholesterol. These results clearly establish that SCAP is required for production of active SREBP-1 and SREBP-2 in animals. Interestingly, mRNA levels of both SREBPs were also dramatically decreased. The promoter specific to the $1 \mathrm{c}$ isoform of SREBP-1 and the single SREBP-2 promoter are both autoactivated by SREBPs in tissue culture studies (Sato et al. 1996; Amemiya-Kudo et al. 2000). The extremely low levels of SREBP mRNAs in the studies of Matsuda et al. (2001) showed that this autoregulation of transcription is critically dependent on SCAP-dependent processing of SREBP.

The role of SCAP in feedback regulation by sterols and insulin was analyzed through a series of feeding studies. It has been known for two decades that when a bile acid sequestrant such as cholestipol and a statin class of 3-hydroxy-3-methylglutaryl coenzyme A (HMG CoA) reductase inhibitor are added to normal chow, it mimics a sterol-depleted state (Brown and Goldstein 1980). Animals respond by increasing expression of proteins required for cholesterol uptake and biosynthesis in the liver. This dietary manipulation resulted in increased processing of the SREBP-2 precursor and decreased processing of SREBP-1 (Sheng et al. 1995). In SCAP-less liver mice, this treatment had no effect on expression of SREBP target genes, which remained very low, showing that SCAP is required to provide at least the minimal levels of SREBP necessary to initiate the response to sterol depletion in animal livers. In fact, the levels of membrane-bound SREBP precursors were also barely detectable before or after treatment with the two compounds. 
Studies over the last few years have revealed that cholesterol and fatty acid metabolism are coregulated by the SREBPs and SREBP-1c is a key activator of lipogenic gene expression in response to insulin signaling in liver (Horton and Shimomura 1999; Osborne 2000). To evaluate whether this process is altered in the SCAP-less liver mouse, Matsuda et al. (2001) adopted a fasting/refeeding regimen to evaluate the effects of insulin on liver lipogenesis. Control animals responded normally with a decrease in lipogenic genes during fasting and a dramatic activation upon refeeding a high-carbohydrate diet. Lipogenic gene expression was already quite low in the mutant animals before the fast and there was little effect of fasting/refeeding. Thus, when SCAP is absent, there are insufficient amounts of processed SREBPs to initiate the program of lipogenesis.

In addition to directly monitoring lipogenic gene activation, liver lipids were also measured along with serum levels of lipids, glucose, and insulin. As expected, serum triglycerides rose upon fasting due to mobilization from adipose tissue stores and was similar in normal and SCAP-less animals. The serum level of glucose falls normally during a fast; this was observed in the current studies in which the glucose levels in control animals fell by $63 \%$ compared with the non-fasted state. However, in the SCAP-less animals, serum glucose levels fell only by $24 \%$. Liver glucose production through gluconeogenesis normally increases upon fasting to compensate for the loss of dietary sugar (Kraus-Friedman 1984). The relatively higher levels of serum glucose in fasted SCAP-less animals may be due to increased gluconeogenesis. Whether this is the source of the increased glucose and whether it is directly a result of low SREBP levels is not yet clear. However, it is intriguing to speculate that because SREBPs are key activators of lipogenesis, they may be negative regulators of key gluconeogenic genes in liver, and this regulation would also be lost in the SCAP-less animal.

Because of the low mRNA levels for the SREBPs and the fact that SCAP appears to stabilize the membranebound precursors (Rawson et al. 1999), there was a surprisingly very low level of expression of even the membrane-bound SREBPs in the control fed SCAP-less animals. Thus, from the animal feeding experiments, it is difficult to distinguish between whether SCAP is only required to provide basal levels of SREBPs that initiate responses to sterol deprivation and insulin signaling or whether SCAP is also a direct target of the regulation. That SCAP is an important component of the regulatory response to sterols is supported by studies in cultured cells in which SCAP movement from the ER to the golgi is increased upon sterol deprivation (Nohturfft et al. 1999). However, there is no supporting evidence that SCAP is a direct regulatory target of insulin signaling. This question may be approached in the future by analyzing transgenic animals that express the precursor SREBPs under the control of a promoter that is not subject to autoregulation.

Elucidation of the elegant mechanism and key proteins in the SCAP-SREBP pathway has been accomplished through experiments performed in both cultured cells and in transgenic mice. In cultured cells, it is possible to totally manipulate the cellular nutritional environment, conditions that are impossible to reproduce in animal feeding studies. Thus, in cultured cells, it is possible to accentuate fundamental molecular events in the regulatory process. These experiments are essential to establish the mechanism, and in combination with totally in vitro experiments, they can precisely identify individual components of a multistep regulatory process. However, these experiments provide only a limited view of the complexities involved, because in whole animals, interacting regulatory pathways can converge at critical points to prevent intolerable swings in metabolic flux. This metabolic compensation can mask the involvement of important regulatory events that operate normally behind the scenes. Because of the complexities of metabolic compensation, simple feeding studies performed in animals can miss important aspects of regulatory pathways that become apparent only by drastic measures in which key proteins are either overexpressed or their genes are knocked out.

For example, the idea that cholesterol metabolism and fatty acid biosynthesis were coregulated was not significantly appreciated until relatively recently. In fact, in early studies that documented feedback regulation of cholesterol biosynthesis by dietary cholesterol, incorporation of labeled precursors into fatty acids was used as a negative control for the specificity of putative regulatory sterols on cholesterol synthesis (Gould 1951; Langdon and Bloch 1953). We now know that cholesterol and fatty acid metabolism are coregulated by SREBPs, and the reason this was not appreciated earlier is that cholesterol biosynthesis is regulated through transcriptional regulation by the SREBPs, a relatively slow-acting but stable regulatory process. High levels of sterols also result in increased degradation of HMG CoA reductase enzyme, which has a rapid effect on inhibiting precursor flux through the pathway (Gil et al. 1985). The early studies probably accentuated regulation mediated through the degradation of HMG CoA reductase and de-emphasized or missed the SREBP regulation.

The fundamental properties of the SCAP-SREBP regulatory cascade have been identified relatively quickly, which underscores the power of combining modern methods of somatic cell genetics, in vitro biochemistry, and transgenic animal studies to tackle a complex problem in a systematic fashion. Several key proteins in this regulatory network have now been identified, and the mechanism of regulation is also becoming well characterized. However, as always, there are still important questions that remain. For example, how is the exit of the SCAP-SREBP complex from the ER regulated by intracellular sterol levels? Also, as SCAP is involved in escorting both SREBP-1 and SREBP-2 from the ER, what is the molecular basis for the differential regulation of SREBP-1 and SREBP-2 processing in animal livers (Sheng et al. 1995)? These and other important questions will likely be answered in the near future through the combined approaches that have so far proven to be highly successful and extremely informative. 


\section{Acknowledgments}

I thank the members of my laboratory for their comments on the manuscript. Unfortunately, space limitations prevented direct reference to several important studies.

\section{References}

Amemiya-Kudo, M., Shimano, H., Yoshikawa, T., Yahagi, N., Hasty, A.H., Okazaki, H., Tamura, Y., Shionoiri, F., Iizuka, Y., Ohashi, K., et al. 2000. Promoter analysis of the mouse sterol regulatory element-binding protein-1c gene. J. Biol. Chem. 275: 31078-31085.

Athanikar, J.N. and Osborne, T.F. 1998. Specificity in cholesterol regulation of gene expression by coevolution of sterol regulatory DNA element and its binding protein. Proc. Natl. Acad. Sci. 95: 4935-4940.

Brown, M.S. and Goldstein, J.L. 1980. Multivalent feedback regulation of HMG CoA reductase, a control mechanism coordinating isoprenoid synthesis and cell growth. J. Lipid Res. 21: 505-517.

- 1999. A proteolytic pathway that controls the cholesterol content of membranes, cells, and blood. Proc. Natl. Acad. Sci. 96: 11041-11048.

DeBose-Boyd, R.A., Ou, J., Goldstein, J.L., and Brown, M.S. 2001. Expression of sterol regulatory element-binding protein 1c (SREBP-1c) mRNA in ret hepatoma cells requires endogenous LXR ligands. Proc. Natl. Acad. Sci. 98: 14771482.

Duncan, E., Dave, U.P., Sakai, J., Goldstein, J.L., and Brown, M.S. 1998. Second-site cleavage in sterol regulatory element-protein occurs at transmembrane junction as determined by cysteine panning. I. Biol. Chem. 273: 1780117809.

Duncan, E.A., Brown, M.S., Goldstein, J.L., and Sakai, J. 1997. Cleavage site for sterol-regulated protease localized to a leuser bond in the lumenal loop of sterol regulatory element binding protein-2. J. Biol. Chem. 272: 12778-12785.

Edwards, P.E. and Ericsson, J. 1999. Steroids and isoprenoids: Signaling molecules derived from the cholesterol biosynthetic pathway. Annu. Rev. Biochem. 68: 157-185.

Edwards, P.E., Tabor, D., Kast, H.R., and Venkateswaran, A. 2000. Regulation of gene expression by SREBP and SCAP. Biochem. et Biophys. 1529: 103-113.

Gil, G., Faust, J.R., Chin, D.J., Goldstein, J.L., and Brown, M.S. 1985. Membrane-bound domain of HMG CoA reductase is required for sterol-enhanced degradation of the enzyme. Cell 41: 249-258.

Gould, R.G. 1951. Lipid metabolism and atherosclerosis. Am. I. Med. 11: 209-227.

Horton, J.D. and Shimomura, I. 1999. Sterol regulatory element binding proteins: Activators of cholesterol and fatty acid biosynthesis. Curr. Opin. Lipidol. 10: 143-150.

Horton, J.D., Bashmakov, Y., Shimomura, I., and Shimano, H. 1998a. Regulation of sterol regulatory element binding proteins in livers of fasted and refed mice. Proc. Natl. Acad. Sci. 95: 5987-5992.

Horton, J.D., Shimomura, I., Brown, M.S., Hammer, R.E., Goldstein, J.L., and Shimano, H. 1998b. Activation of cholesterol synthesis in preference to fatty acid synthesis in liver and adipose tissue of transgenic mice overproducing sterol regulatory element-binding protein-2. J. Clin. Invest. 101: 23312339.

Hua, X., Yokoyama, C., Wu, J., Briggs, M.R., Brown, M.S., Goldstein, J.L., and Wang, X. 1993. SREBP-2, a second basic-helixloop-helix-leucine zipper protein that stimulates transcrip- tion by binding to a sterol regulatory element. Proc. Natl. Acad. Sci. 90: 11603-11607.

Hua, X., Nohturfft, A., Goldstein, J.L., and Brown, M.S. 1996a. Sterol resistance in $\mathrm{CHO}$ cells traced to point mutation in SREBP cleavage-activating protein. Cell 87: 415-426.

Hua, X., Sakai, J., Brown, M.S., and Goldstein, J.L. 1996b. Regulated cleavage of sterol regulatory element binding proteins requires sequences on both sides of the endoplasmic reticulum membrane. J. Biol. Chem. 271: 10379-10384.

Kim, J.B., Spotts, G.D., Halvorsen, Y.-D., Shih, H.-M., Ellenberger, T., Towle, H.C., and Spiegelman, B.M. 1995. Dual DNA binding specificity of ADD1/SREBP-1 controlled by a single amino acid in the basic helix-loop-helix domain. Mol. Cell. Biol. 15: 2582-2588.

Kim, J.B., Sarraf, P., Wright, M., Yao, K.M., Mueller, E., Solanes, G., Lowell, B.B., and Speigelman, B.M. 1998. Nutritional and insulin regulation of fatty acid synthetase and leptin gene expression through ADD1/SREBP-1c. J. Clin. Invest. 101: $1-9$.

Kraus-Friedman, N. 1984. Hormonal regulation of hepatic gluconeogenesis. Physiol. Rev. 64: 170-259.

Langdon, R.G. and Bloch, K. 1953. The effects of some dietary additions on the synthesis of cholesterol from acetate in vitro. J. Biol. Chem. 202: 77-81.

Lane, S., Zhou, S., Pan, T., Dai, Q., and Liu, H. 2001. Cph2, a member of bHLH proteins, is a regulator of hyphal development in Candida albicans. Mol. Cell. Biol. (in press).

Matsuda, M., Korn, B.S., Hammer, R.E., Moon, Y.-A., Kumuro, R., Horton, J.D., Goldstein, J.L., Brown, M.S., and Shimomura, I. 2001. SERBP cleavage-activating protein (SCAP) is required for increased lipid synthesis in liver induced by cholesterol deprivation and insulin elevation. Genes \& Dev. 15: 1206-1216.

Miserez, A.R., Cao, G., Probst, L., and Hobbs, H.H. 1997. Structure of the human gene incoding sterol regulatory element binding protein 2 (SREBF2). Genomics 40: 31-40.

Nishi, K., Park, C.S., Pepper, A.E., Eichinger, G., Innis, M., and Holland, M.J. 1995. The GCR1 requirement for yeast glycolytic gene expression is suppressed by dominant mutations in the SGC1 gene, which encodes a novel basic-helix-loophelix protein. Mol. Cell. Biol. 15: 2646-2653.

Nohturfft, A., DeBose-Boyd, R.A., Scheek, S., Goldstein, J.L., and Brown, M.S. 1999. Sterols regulate cycling of SREBP cleavage-activating protein (SCAP) between endoplasmic reticulum and Golgi. Proc. Natl. Acad. Sci. 96: 11235-11240.

Nohturfft, A., Yabe, D., Goldstein, J.L., and Brown, M.S. 2000. Regulated step in choelsterol feedback localized to budding of SCAP from ER membranes. Cell 102: 315-323.

Osborne, T.F. 2000. Sterol regulatory element binding proteins (SREBPs): Key regulators of nutritional homeostasis and insulin action. J. Biol. Chem. 275: 32379-32382.

Parraga, A., Bellsolell, L., Ferre-D'Amare, A.R., and Burley, S.K. 1998. Co-crystal structure of sterol regulatory element binding protein $1 \mathrm{a}$ at $2.3 \AA$ resolution. Structure 6: 661-672.

Rawson, R.B., Zelenski, N.G., Nijhawan, D., Ye, J., Sakai, J., Hasan, M.T., Chang, T.Y., Brown, M.S., and Goldstein, J.L. 1997. Complementation cloning of S2P, a gene encoding a putative metalloprotease required for intramembrane cleavage of SREBPs. Mol. Cell 1: 47-57.

Rawson, R.B., DeBose-Boyd, R.A., Goldstein, J.L., and Brown, M.S. 1999. Failure to cleave sterol regulatory element binding proteins (SREBPs) causes cholesterol auxotrophy in chinese hamster ovary cells with genetic absence of SREBP cleavage activating protein. I. Biol. Chem. 274: 2854928556.

Sakai, J., Nohturfft, A., Cheng, D., Ho, Y.K., Brown, M.S., and 
Goldstein, J.L. 1997. Identification of complexes between the COOH-terminal domains of sterol regulatory elementbinding proteins (SREBPs) and SREBP cleavage-activating protein. J. Biol. Chem. 272: 20213-20221.

Sakai, J., Rawson, R.B., Espenshade, P., Cheng, D., Seegmiller, A.C., Goldstein, J.L., and Brown, M.S. 1998. Molecular identification of the sterol regulated luminal protease that cleaves SREBPs and controls lipid composition of animal cells. Mol. Cell 2: 505-514.

Sato, R., Inoue, J., Kawabe, Y., Kodama, T., Takano, T., and Maeda, M. 1996. Sterol-dependent transcriptional regulation of sterol regulatory element-binding protein-2. T. Biol. Chem. 271: 26461-26464.

Sheng, Z., Otani, H., Brown, M.S., and Goldstein, J.L. 1995. Independent regulation of sterol regulatory element-binding proteins 1 and 2 in hamster liver. Proc. Natl. Acad. Sci. 92: 935-938.

Shimano, H., Horton, J.D., Shimomura, L., Hammer, R.E., Brown, M.S., and Goldstein, J.L. 1997. Isoform 1c of sterol regulatory element binding protein is less active than isoform 1a in livers of transgenic mice and cultured cells. $J$. Clin. Invest. 99: 846-854.

Shimano, H., Yahagi, N., Amemiya-Kudo, M., Hasty, A.H., Osuga, J., Tamura, Y., Shionoiri, F., Iizuka, Y., Ohashi, K., Harada, K., et al. 1999. Sterol regulatory element-binding protein-1 as a key transcription factor for nutritional induction of lipogenic enzyme genes. J. Biol. Chem. 274: 35832 35839.

Shimomura, L., Shimano, H., Horton, J.D., Goldstein, J.L., and Brown, M.S. 1997. Differential expression of exons 1a and 1c in mRNAs for sterol regulatory element binding protein-1 in human and mouse organs and cultured cells. J. Clin. Invest. 99: 838-845.

Tontonoz, P., Kim, J.B., Graves, R.A., and Spiegelman, B.M. 1993. ADD1: A novel helix-loop-helix transcription factor associated with adipocyte determination and differentiation. Mol. Cell. Biol. 13: 4753-4759.

Towle, H.C., Kaytor, E.N., and Shih, H.M. 1997. Regulation of the expression of lipogenic enzyme genes by carbohydrate. Annu. Rev. Nutr. 17: 405-433.

Wang, X., Sato, R., Brown, M.S., Hua, X., and Goldstein, J.L. 1994. SREBP-1, a membrane-bound transcription factor released by sterol regulated proteolysis. Cell 77: 53-62.

Ye, J., Rawson, R.B., Komuro, R., Chen, X., Dave, U.P., Prywes, R., Brown, M.S., and Goldstein, J.L. 2000. ER stress induces cleavage of membrane-bound ATF6 by the same proteases that process SREBPs. Mol. Cell 6: 1355-1364.

Yokoyama, C., Wang, X., Briggs, M.R., Admon, A., Wu, J., Hua, X., Goldstein, J.L., and Brown, M.S. 1993. SREBP-1, a basichelix-loop-helix-zipper protein that controls transcription of the low density receptor gene. Cell 75: 185-197. 


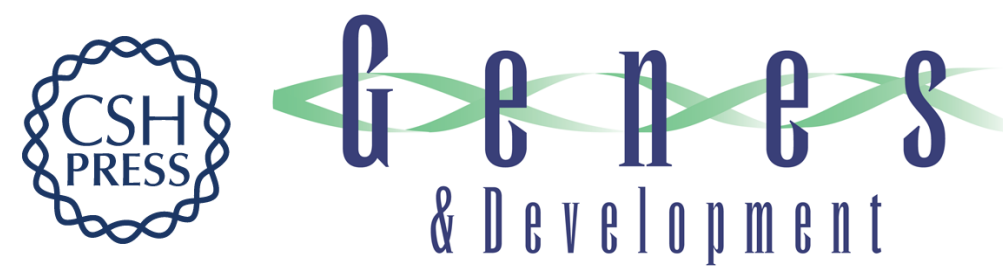

\section{CREating a SCAP-less liver keeps SREBPs pinned in the ER membrane and prevents increased lipid synthesis in response to low cholesterol and high insulin}

Timothy F. Osborne

Genes Dev. 2001, 15:

Access the most recent version at doi:10.1101/gad.916601

\section{References This article cites 40 articles, 22 of which can be accessed free at: http://genesdev.cshlp.org/content/15/15/1873.full.html\#ref-list-1}

\section{License}

Email Alerting
Service

Receive free email alerts when new articles cite this article - sign up in the box at the top right corner of the article or click here.

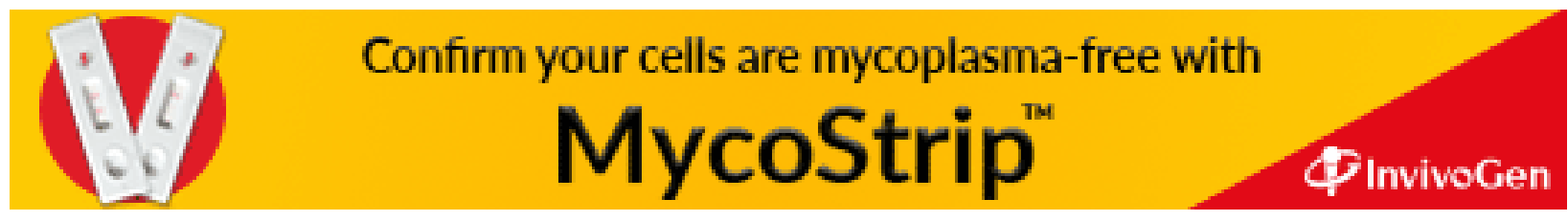

